\title{
Mammazentrum Ostbayern - Ein Kompentenzzentrum zur flächendeckenden optimalen Versorgung von Brustkrebspatientinnen
}

\author{
Doris Augustin \\ Klinikum des Landkreises Deggendorf, Germany
}

Die Zentralisierung und Spezialisierung bezüglich der Diagnostik und Therapie des Mammakarzinoms ist in den letzten Jahren zunehmend politisch gewollt und unter diesem Druck sind zahlreiche Brustzentren entstanden. Das Mammazentrum Ostbayern am Klinikum Deggendorf unterscheidet sich von vielen neueren Brustzentren darin, dass es nicht unter politischem oder wirtschaftlichem Druck in kurzer Zeit entstanden, sondern aus gewachsenen Strukturen hervorgegangen ist. Es handelt sich um eine Institution, die zum Zeitpunkt der Gründung im September 2000 bereits auf mehrjährige interdisziplinäre Erfahrungen zurückblicken konnte und sich den nun gewünschten politischen Anforderungen sehr früh gestellt hat (Tab. 1). Die Besonderheit des Mammazentrums Ostbayern liegt darin, dass es sich um eine seit Jahren gewachsene Einheit handelt, in der alle drei Gründungspart- ner Radiologie, Pathologie und Senologie seit langem mit dem höchsten Qualitätsanspruch interdisziplinär zusammenarbeiten.

Die Gründung einer eigenen Abteilung für Senologie hat hier sicherlich wesentlich zum Erfolg des Konzepts beigetragen. Die Aufgabe der Senologie ist unter anderem die Koordination zwischen Radiologen, Pathologen, Strahlentherapeuten, plastischen Chirurgen und betreuenden Frauenärzten, Hausärzten und Onkologen. Die weiteren Aufgabenbereiche sind die Diagnostik, die operative und onkologische Therapie, die risikoadaptierte Nachsorge, die psychosoziale und gynäkologische Betreuung und das Angebot spezieller Sprechstunden. Hinzu gekommen sind in den letzten Jahren die Betreuung der lückenlosen Datenerfassung und das Qualitätsmanagement.
Tab. 1. Der Weg zum heutigen Mammazentrum Ostbayern

\section{Seit 1996 \\ Seit 1996}

Seit 12/1998

Seit 1998

September 2000

Seit 2000

Seit 2003

Seit Oktober 2003

Seit 2003

September 2004

Herbst 2005

September 2005

Seit Anfang 2006

2006

Ende 2006 ultraschallgesteuerte Stanzbiopsien

in ca. $65-70 \%$ brusterhaltende Therapie

Vakuumstanzbiopsien (Teilnahme an der Multicenterstudie zur Vakuumstanzbiopsie von Frau Prof. Dr. Heywang-Köbrunner)

Teilnahme an klinischen Studien der AGO und GABG (jetzt GBG)

Gründung des Mammazentrums Ostbayern und der Abteilung Senologie

Datenerfassung im onkologischen Dokumentationssystem ODS EASY der Fa. Asthenis

komplette Erfassung aller Daten einschließlich Nachsorge und Rezidive

Assessmentcenter für das Bayerische Mammographiescreening

Kooperationspartner des Studienzentrums der TU-München Rechts der Isar, seither auch Bestimmung der Proteasen upA und PAI-1 am Tumorgewebe

Zertifizierung durch die DKG und DGS und LGA nach nur viermonatiger Vorbereitungszeit

Kooperationsvereinbarung mit dem WBC und BBC zum externen Benchmarking und zum externen

Datenmonitoring

5-jähriges Bestehen des Mammazentrums

Teilnahme an Europäischer Studie zur interstitiellen Brachytherapie (Teilbrustbestrahlung) in

Zusammenarbeit mit der Strahlentherapie Barmherzige Brüder in Regensburg

Gründung einer eigenen Strahlentherapie am Klinikum Deggendorf Etablierung einer

humangenetischen Sprechstunde durch entsprechende Kooperationsvereinbarung

Assessmentcenter für Niederbayern im bundesweiten Mammographie-Screening

\section{KARGER}

Fax +497614520714

E-mail Information@Karger.de

www.karger.com

\section{(C) 2006 S. Karger GmbH, Freiburg}

Accessible online at:

www.karger.com/brc
Doris Augustin

Mammazentrum Ostbayern

Klinikum des Landkreises Deggendorf

Postfach 1526, 94455 Deggendorf, Germany

Tel. +49 991 380-3171, Fax -3177

E-mail doris.augustin@klinikum-deggendorf.de 


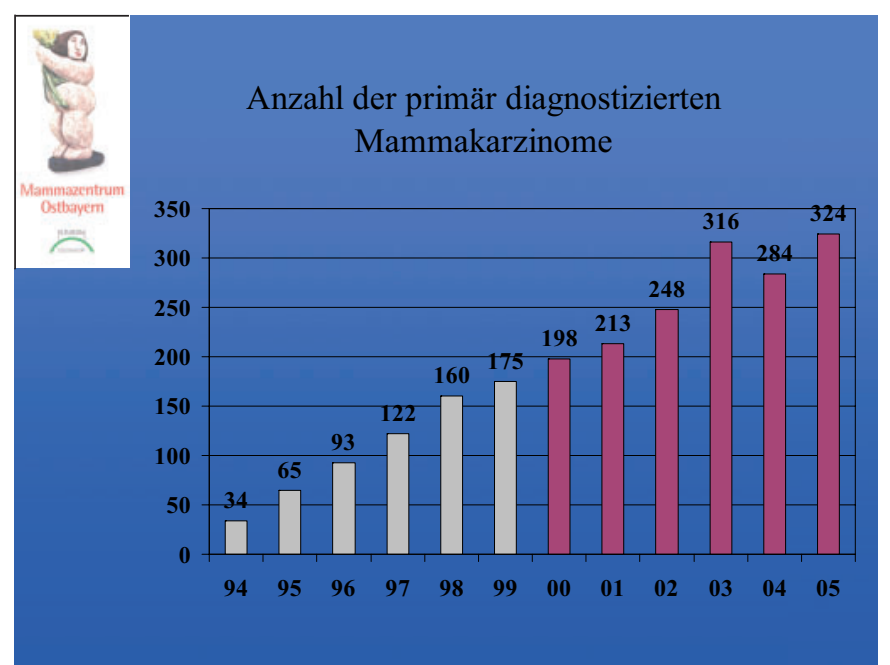

Abb. 1. Anzahl der primär diagnostizierten Mammakarzinome im Mammazentrum Ostbayern.

Die ambulante Versorgung der Patienten findet unter einem Dach mit kurzen Wegen und allerhöchstem Patientenkomfort statt.

Die Patientin bleibt vom Zeitpunkt der Verdachtsdiagnose bis zum Ende der Nachsorge oder im Falle der Metastasierung bis zum Tod in der Betreuung des Kernteams von spezialisierten Ansprechpartnern. Diese Kontinuität erstreckt sich vom diagnostischen und therapeutischen Bereich bis zur pflegerischen und psychoonkologischen Betreuung. Nicht selten geht die Betreuung der Familie und Angehörigen über den Tod hinaus. Die Kontinuität der Ansprechpartner wird sowohl von Betroffenen als auch von den überweisenden Ärzten sehr geschätzt.

Der Auftrag des Mammazentrums Ostbayern besteht in der optimalen und flächendeckenden Versorgung der Patientinnen mit der Diagnose Brustkrebs in dieser Region. Dies geschieht durch die seit der Gründung des Zentrums bestehenden Kooperation mit der Bayerischen Krebsgesellschaft. Durch die gemeinsame intensive Aufklärungs- und Öffentlichkeitsarbeit wird erreicht, dass immer mehr Frauen der Früherkennung, aber auch der optimalen Therapie zugeführt werden. In den letzten drei Jahren sind so jährlich etwa 300 primäre Mammakarzinome diagnostiziert worden (Abb. 1) und zirka $130 \mathrm{~Pa}-$ tientinnen mit metastasiertem Mammakarzinom betreut worden. Dabei nehmen 26\% der Patientinnen an Studien teil.

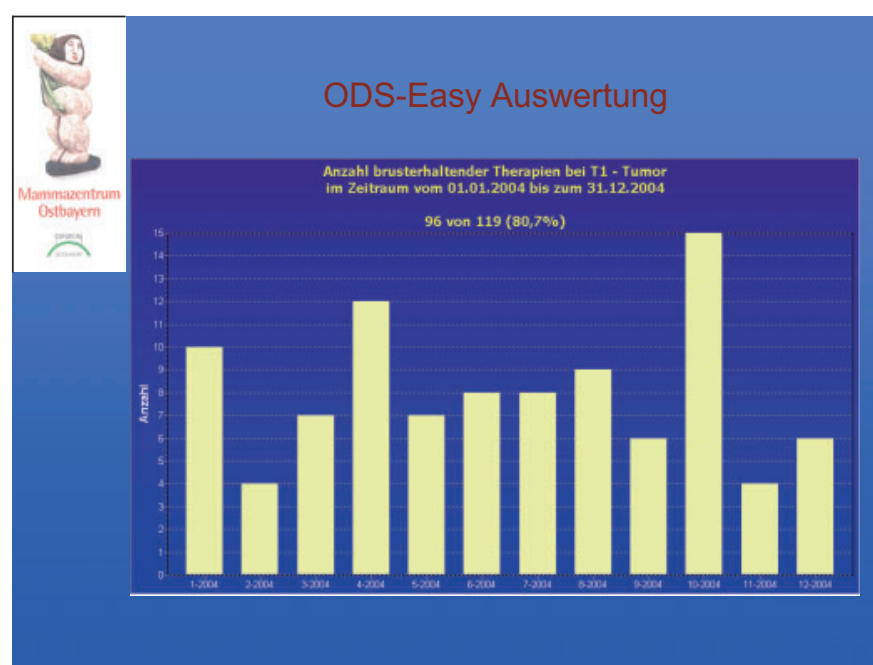

Abb. 2. Anzahl brusterhaltender Therapien bei T1-Tumor im Jahr 2004.

Der langjährige Aufbau eines Netzwerks mit verschiedenen Kooperationspartnern wie die vier strahlentherapeutischen Abteilungen im gesamten Einzugsgebiet des Zentrums, eines plastischen Chirurgen und jetzt einer Humangenetikerin sowie die Anbindung an das Studienzentrum der TU München ermöglichen unseren Patientinnen Zugang zur Diagnostik und Therapie auf allerhöchstem Niveau. So nahmen ein erheblicher Teil unserer dafür geeigneten Patientinnen an der internationalen HERA-Studie teil, ermöglicht durch die Kooperation mit dem Studienzentrum.

Seit Anfang des Jahres werden Patientinnen, die dafür geeignet sind, in eine europäische Studie zur interstitiellen Brachytherapie (Teilbrustbestrahlung) eingebracht. Das Verfahren reduziert die Strahlenbelastung und verkürzt die Behandlungsdauer um mehrere Wochen. Die Studie ist bei unseren Patientinnen gut aufgenommen worden.

Auch die Ergebnisse des externen Benchmarkings durch das WBC im bundesweiten Vergleich mit 300 weiteren Kliniken zeigt, das wir unserem hohen Qualitätsanspruch gerecht werden. So konnten 2004 80,7\% der Patientinen mit T1-Tumor brusterhaltend behandelt werden (Abb. 2).

Durch die Schaffung des Mammazentrums Ostbayern wird allen Patientinnen dieser vorwiegend ländlichen Region der Zugang zu modernster Diagnostik und Therapie ermöglicht. 